\title{
EFICIÊNCIA DAS AGÊNCIAS BANCÁRIAS TRADICIONAIS SOB A ÓTICA DA ANÁLISE ENVOLTÓRIA DE DADOS
}

\author{
Marcos Paulo Albarello Friedrich \\ Fundação Universidade Regional de Blumenau - FURB \\ Rua Siqueira Campos, 832 - Porto Alegre - RS - Brasil - CEP 90010-000 \\ mfriedrich@furb.br \\ Nelson Hein \\ Fundação Universidade Regional de Blumenau - FURB \\ Rua Antônio da Veiga, 140, Blumenau, SC - Brasil - CEP 89010-971 \\ hein@furb.br \\ Adriana Kroenke Hein \\ Fundação Universidade Regional de Blumenau - FURB \\ Rua Antônio da Veiga, 140, Blumenau, SC - Brasil - CEP 89010-971 \\ akroenke@furb.br
}

\begin{abstract}
RESUMO
O mercado financeiro brasileiro tradicional, formado por agências bancárias espalhadas pelo país, vem tendo sua eficiência questionada frente ao avanço das fintechs que operam sem lojas físicas e com custo inferior. Diante disso, essa pesquisa tem por objetivo avaliar a eficiência de agências bancárias a partir da abordagem de rentabilidade. Para atendimento ao objetivo do estudo analisou-se uma rede de 56 agências bancárias, em 49 municípios. Os dados considerados foram: número de empregados e de clientes, despesas totais, PIB do município, número de agências concorrentes, margem de contribuição por produto, de tarifas e média por cliente, receita líquida. Utilizou-se o método da Análise Envoltória de Dados para avaliação da eficiência das agências com foco em produto e retornos variáveis (BCCVRS output). Os resultados evidenciaram que 22 agências foram consideradas eficientes e 34 não eficientes, indicando menor nível de preparação para competir com os novos players do mercado. Por fim, identificou-se agências com eficiência inferior a $70 \%$ para análise pontual e desenvolvimento de estratégias para melhoria tendo como benchmark o melhor desempenho entre as agências avaliadas.
\end{abstract}

Palavra-chave: Mercado financeiro. Eficiência bancária. Análise envoltória de dados.

\begin{abstract}
The traditional Brazilian financial market, made up of bank branches throughout the country, has been questioning its efficiency in view of the advance of fintechs that operate without physical stores and at a lower cost. Given this, this research aims to evaluate the efficiency of bank branches from the approach of profitability. To meet the objective of the study, a network of 56 bank branches in 49 municipalities was analyzed. The data considered were: number of employees and customers, total expenses, municipal GDP, number of competing agencies, contribution margin per product, tariffs and average per customer, net revenue. The Data Envelopment Analysis method was used to evaluate the efficiency of the agencies focused on product and variable returns (BCC-VRS output). The results showed that 22 branches were considered efficient and 34 not efficient, indicating a lower level of
\end{abstract}


preparation to compete with the new players in the market. Finally, agencies with less than $70 \%$ efficiency were identified for spot analysis and development of improvement strategies based on the best performance among the evaluated agencies.

Keyword: Financial Market. Bank efficiency. Data envelopment analysis.

\section{Como Citar:}

FRIEDRICH, Marcos Paulo Albarello; Hein,Nelson; Hein, Adriana Kroenke. Eficiência Das Agências Bancárias Tradicionais Sob A Ótica Da Análise Envoltória De Dados. In: SIMPÓSIO DE PESQUISA OPERACIONAL E LOGÍSTICA DA MARINHA, 19., 2019, Rio de Janeiro, RJ. Anais [...]. Rio de Janeiro: Centro de Análises de Sistemas Navais, 2019.

\section{INTRODUÇÃO}

Observa-se que o mercado financeiro brasileiro tradicional, formado por agências bancárias espalhadas pelo país, vem passando por uma revolução nos últimos anos em função da instabilidade causada pelo cenário político-econômico e devido ao ingresso de novos players, como empresas não-bancárias e fintechs, que têm apresentado novos modelos de negócios baseados em soluções tecnológicas inovadoras.

Segundo Fintechlab (2017), em 2015 o mercado brasileiro apresentava 54 fintechs enquanto em novembro de 2017 esse número cresceu para 332, sendo que o investimento no último ano foi de mais de $\mathrm{R} \$ 1,028$ bilhão. Oliveira e Marques (2017) destacam que esse avanço se deve, principalmente, ao baixo custo de funcionamento dessas empresas e a elevada aceitação do mercado. Por outro lado, os bancos tradicionais apresentam custo mais elevado quando comparados às fintechs devido à instalação de agências, empregados para atendimento presencial, material de expediente etc. (FEBRABAN, 2016). Diante desse cenário, observa-se uma reação intensa desses bancos para manterem-se competitivos: fechamentos de agências, planos de demissão voluntária, planos de aposentadoria incentivada e, principalmente, parcerias com startups de tecnologia (FEBRABAN, 2016; SOUSA, MACEDO, 2009).

Todo esse movimento promovido pelos bancos tradicionais tem como pano de fundo a eficiência de seu negócio e reforça a importância de sua mensuração como forma de apoio à tomada de decisão (DOUMPOS, COHEN, 2014; LIU, 2010). Os critérios definidores da eficiência de uma unidade, os quais definem ou não sua continuidade, por exemplo, estão mudando e exigindo que as instituições tradicionais olhem de maneira diferenciada para seus indicadores de modo a realinhar estratégias e identificar pontos ainda ineficientes.

Diante desse cenário de alta competitividade, métodos de análise de eficiência monocritério, com enfoque apenas em índices financeiros, já não permitem uma resposta à altura dos resultados necessários para sobrevivência no mercado (CERETTA, NIEDERAUER, 2001). Nesse sentido, diversos estudos como Ceretta e Niederauer (2001), Périco et al. (2008), Faria e Paula (2009), Sahin et al. (2016), Zhong e Zhao (2016) e Comelli, Kroenke e Hein (2017) foram realizados com intuito de analisar a eficiência de instituições financeiras. Ressalta-se que Carneiro, Pereira e Macoris (2016) realizaram uma pesquisa relacionando as diversas formas de abordagem para medida de eficiência e argumentam que, apesar de não haver consenso na literatura, três abordagens são mais comuns: a de intermediação, de produção e de rentabilidade.

Sendo assim, a questão que norteia este estudo é: Qual o nível de eficiência das agências pertencentes a um banco tradicional com base na abordagem rentabilidade e considerando-se uma avaliação multicritério? Para responder a essa questão, tem-se como objetivo avaliar a eficiência de agências bancárias a partir da abordagem da rentabilidade. Adota- 
se a abordagem da rentabilidade que, segundo Fethi e Pasiouras (2010), considera como inputs os componentes de custos dos bancos e como outputs os componentes da receita. Além disso, considera-se variáveis exógenas como PIB e número de concorrentes .

Busca-se, portanto, contribuir para o avanço e melhoraria da análise da eficiência de bancos tradicionais permitindo a comparação entre as unidades e possibilitando aos gestores uma análise para possível adequação de suas estratégias visando o alcance da eficiência. Nesse sentido, o estudo justifica-se, pois, o setor financeiro brasileiro possui um mercado de capitais tímido e pouco desenvolvido se comparado com países de primeiro mundo e isso faz com que os bancos tradicionais sejam os principais fomentadores do desenvolvimento econômico e social (CARVALHO et al., 2016).

As análises foram realizadas por meio da Análise Envoltória de Dados (DEA) por ser uma ferramenta bastante utilizada para avaliar a eficiência de organizações, principalmente por permitir identificar folgas e indicadores que ainda podem ser melhorados (ABLANEDO-ROSAS et al., 2010; ZHU, 2000). Por ser um método multicritério, a utilização dessa metodologia se justifica, permitindo uma análise financeira que hierarquiza as unidades analisadas em relação ao melhor desempenho dentro do grupo e apresenta-se como alternativa a métodos tradicionais de análise (DOUMPOS, COHEN, 2014).

\section{ANÁLISE ENVOLTÓRIA DE DADOS}

A formulações iniciais da Análise Envoltória de Dados (DEA) têm início junto com os primeiros registros da teoria microeconômica da produção, por volta do século XVIII. Contribuições foram importantes depois disso, como os trabalhos de John von Neumann em 1935, que desenvolveu um modelo de programação de expansão da economia, e de George Bernar Dantizig, que reestruturou o problema de programação linear desenvolvendo o método chamado Simplex, em 1951 (FERREIRA, GOMES, 2009).

Outros trabalhos como Koopmans (1951) e Debreu (1951) também foram importantes nessa fase inicial, mas os mentores desse método atribuem a contribuição fundamental para criação da DEA ao trabalho de Farrel (1957), intitulado The Measurement of Productive Efficiency, no qual o autor afirma que, uma vez definida a fronteira de eficiência, a medida de ineficiência de uma unidade produtiva (DMU) corresponde a distância entre o nível de produção observado e a fronteira de eficiência (ARAUJO, CARMONA, 2002). Apesar disso, suas propostas são consideradas limitadas, pois apesar de combinar insumos (inputs), utilizava apenas um produto (output) o que não atende a situações reais com grandes conjuntos de dados.

Somente com o trabalho de Charnes, Cooper e Rhodes (1978) que o termo "Análise Envoltória de Dados" surgiu e se popularizou. Esse método tem como objetivo avaliar a eficiência relativa, isto é, em comparação ao melhor padrão (benchmark) de um grupo de unidades produtivas tomadoras de decisão (Decision Making Units - DMUs), alterando a definição de fronteira de eficiência.

A literatura apresenta duas abordagens para fronteira de eficiência: a paramétrica e a não-paramétrica. A primeira define a fronteira através do método dos mínimos quadrados (método de regressão) e, portanto, baseia-se em medidas de tendência central (Figura 1). Já na não-paramétrica, a eficiência é medida de acordo com as a performance das demais unidades do grupo, sendo que a melhor delas será considerada a eficiente e todas as demais estarão igual ou abaixo desse valor. Logo, baseia-se nos valores extremos e não na média central (ARAÚJO, CARMONA, 2002). 
Figura 1: Comparação entre as curvas formadas pela fronteira de eficiência (DEA) e a linha de regressão

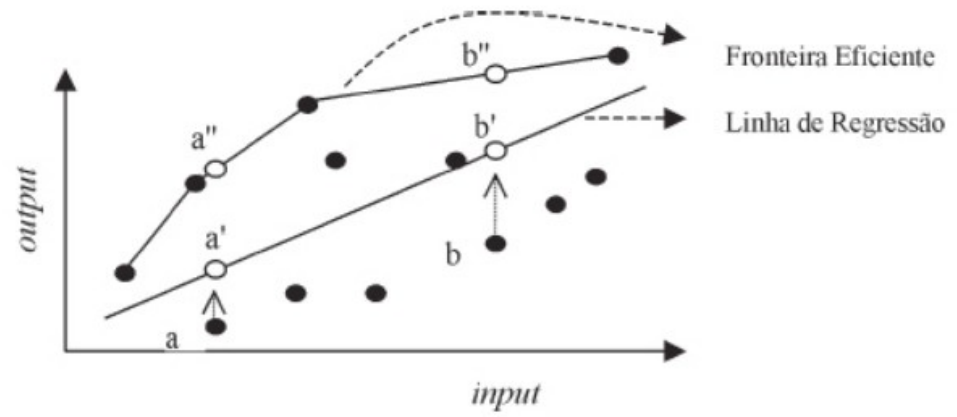

Fonte: adaptado de Ceretta e Niederauer (2001).

Deste modo, o Data Envelopment Analysis - DEA ou Análise Envoltória de Dados é um método não paramétrico que estende a análise de eficiência de índices de output/input únicos para a situação de múltiplos outputs e múltiplos inputs. A eficiência de cada DMU em análise é mensurada em relação às outras do grupo e, portanto, enquanto técnicas estatísticas comuns avaliam eficiência em relação a um produtor médio, a técnica de DEA é um método de valores extremos e compara cada produtor apenas com o melhor produtor de um determinado grupo (ARAÚJO, CARMONA, 2002; CASADO, SOUZA, 2007; FERREIRA, GOMES, 2009). O fato de não depender de inferências estatísticas gera uma vantagem operacional do DEA em relação a outras técnicas (CARNEIRO, PEREIRA, MACORIS, 2016).

O DEA define o que se chama de curva de eficiência relativa ou de máxima produtividade, a partir da avaliação da melhor relação input/output apresentada no grupo. Dessa forma, as DMUs que obtiveram a melhor performance na relação insumos/produtos são chamadas de eficientes e posicionadas sobre a curva. As outras DMUs são chamadas de não eficientes e posicionadas abaixo da curva (BARBOSA; MACEDO, 2008; MENDONÇA, SOUZA, CAMPOS, 2016).

Dentre as técnicas existentes para mensurar eficiência, adota-se a Análise Envoltória de Dados neste estudo, pois, além de ser recomendada por Cooper, Seiford e Zhu (2011), esta técnica possui grande aceitação na comunidade acadêmica, tendo sido utilizada 3183 já entre 1978 e 2001 (TAVARES, 2002) e com uma extensa lista de vantagens e desvantagens compara à regressão (THANASSOULIS, 1993). O DEA considera uma fronteira extrema de eficiência e, somado a isso, calcula a folga de ineficiência que cada unidade possui, permitindo identificar em que insumos ou produtos a unidade precisa melhorar sua produção (CARNEIRO, PEREIRA, MACORIS, 2016).

O DEA apresenta quatro variações básicas: o modelo CCR-CSR (retornos fixos) com orientação para o insumo ou para o produto e, o modelo BCC-VSR, que pode ter orien tação para insumo ou para produto, mas apresenta retornos variáveis. Ferreira e Gomes (2009) afirmam que os retornos de escala representam a relação entre as varrições dos inputs e outputs, isto é, quando eles aumentam e diminuem em uma mesma proporção os retornos são constantes, quando não existe relação proporcional, diz-se que os retornos são variáveis.

No modelo Charnes, Cooper e Rhodes - CCR (1978), a orientação para insumos busca eficiência em reduzir-se os níveis de inputs mantendo constante o produto (output). Já a orientação para o produto, busca a ampliação dos outputs, mantendo os inputs constantes, considerando os retornos constantes (PÉRICO et al. (2008), conforme Quadro 1.

\section{Quadro 1 - modelos CCR}




\begin{tabular}{|c|c|}
\hline Modelo CCR -Orientação Input & Modelo CCR -Orientação Output \\
\hline $\begin{array}{c}\text { Maximizar } h_{k}=\sum_{r=1} u_{r} y_{r k} \\
\text { Sujeito a: } \\
\sum_{i=1}^{n} v_{i} x_{i k}=1 \\
\sum_{r=1}^{m} u_{r} y_{r j}-\sum_{i=1}^{n} v_{i} x_{i j} \leq 0 \\
u_{i}, v_{i} \geq 0 \\
\text { Considerando: } \\
y=\text { outputs; } x=\text { inputs } \\
u, v=\text { pesos } \\
r=1, \ldots, m ; i=1, \ldots, n ; j=1, \ldots, N\end{array}$ & $\begin{array}{c}\text { Minimizar } h_{k}=\sum_{i=1} v_{i} x_{i k} \\
\text { Sujeito a: } \\
\sum_{r=1}^{m} u_{r} y_{r k}=1 \\
\sum_{r=1}^{m} u_{r} y_{r j}-\sum_{i=1}^{n} v_{i} x_{i j} \leq 0 \\
u_{r}, v_{i} \geq 0 \\
\text { Considerando: } \\
y=\text { outputs; } x=\text { inputs } \\
u, v=\text { pesos } \\
r=1, \ldots, m ; i=1, \ldots, n ; j=1, \ldots, N\end{array}$ \\
\hline
\end{tabular}

Fonte: Adaptado de Périco et al. (2008).

O modelo BCC criado por Banker, Charnes e Cooper (1984) permite distinguir eficiência técnica de eficiência de escala estimando uma eficiência técnica e identificando se há ganhos pela variação da escala (retornos variáveis). Os modelos são apresentados no Quadro 2.

\section{Quadro 2 - modelos BCC}

\begin{tabular}{|c|c|}
\hline Modelo BCC -Orientação Input & Modelo BCC -Orientação Output \\
\hline $\begin{array}{c}\text { Maximizar } \sum_{r=1}^{m} u_{r} y_{r k}-u_{k} \\
\text { Sujeito a: } \\
\sum_{r=1}^{n} v_{i} x_{k i}=1 \\
\sum_{r=1}^{m} u_{r} y_{r j}-\sum_{i=1}^{n} v_{i} x_{i j}-v_{k} \leq 0 \\
u_{r} v_{i} \geq 0 \\
\text { Considerando: } \\
y=\text { outputs; } x=\text { inputs } \\
u, v=\text { pesos } \\
r=1, \ldots, m ; i=1, \ldots, n ; j=1, \ldots, N\end{array}$ & $\begin{array}{c}\text { Minimizar } \sum_{i=1}^{n} v_{i} x_{k i}+v_{k} \\
\text { Sujeito a: } \\
\sum_{r=1}^{m} u_{r} y_{r k}=1 \\
\sum_{r=1}^{m} u_{r} y_{r k}=1-\sum_{i=1}^{n} v_{i} x_{j r}-v_{k} \leq 0 \\
u_{r} v_{i>0} \\
\text { Considerando: } \\
y=\text { outputs; } x=\text { inputs } \\
u, v=\text { pesos } \\
r=1, \ldots, m ; i=1, \ldots, n ; j=1, \ldots, N\end{array}$ \\
\hline
\end{tabular}

Fonte: Adaptado de Périco et al. (2008).

Desse modo, esse modelo permite a utilização de DMU's com diferentes tamanhos, porém, como esse modelo forma uma superfície convexa de planos em intersecção envolvendo os dados de uma maneira mais compacta que o método CCR, se uma DMU é eficiente no método CCR-CSR ela também será no BCC-VRS (Quadro 2) (COMELLI et al., 2017; FERREIRA, GOMES, 2009).

\section{PROCEDIMENTOS METODOLÓGICOS}


O presente estudo possui natureza descritiva, multi-caso, com abordagem quantitativa dos dados mediante aplicação da técnica de Análise Envoltória de Dados para calcular escores de eficiência das agências bancárias de um banco tradicional da região Sul do Brasil.

Segundo Périco et al. (2008), a primeira etapa do DEA é a seleção das unidades para análise, sendo que estas devem possuir mesmos objetivos, mesmas condições de trabalho e variáveis de análise, isto é, devem ser homogêneas. Desse modo, o objeto de estudo consiste em 56 agências situadas em 49 diferentes municípios de uma mesma região do Rio Grande do Sul (RS), de uma instituição que possui 527 agências no mesmo estado. Escolheu-se tal instituição por ser um banco tradicional que abrange aproximadamente $98 \%$ dos municípios dessa região e, optou-se por analisar agências da mesma região por se entender atuarem em municípios cuja base da economia é semelhante. Para análise do contexto em que as unidades estão inseridas utilizou-se variáveis que aliam os dados contábeis ao mercado de atuação conforme sugerem Comelli et. al (2017), ou seja, os inputs considerados foram: número de empregados por agência (EMP) e média anual de despesas totais (DPTOT). Além desses, considerou-se também como inputs dados do ambiente de negócios como o PIB dos municípios (PIB) cujos dados foram extraídos da página da Fundação de Economia e Estatística do RS (2014), as informações acerca do número de bancos que atuam no município (BCMUN) e número total de agências concorrentes (AGCON), as quais foram coletadas diretamente no site do Banco Central do Brasil (em dezembro 2017 com referência ao mês de outubro 2017).

Como outputs, foram considerados: margem de contribuição com produtos (MCPROD), margem de contribuição com tarifas (MCTAR), média anual da receita líquida mensal (RLIQ), número de clientes (CLI), margem de contribuição média por cliente (MCMED), tendo como período base o mês de outubro de 2017. A coleta ocorreu através da análise de documentos internos em quatro visitas as instituições realizadas entre 15 e 27 de novembro de 2017.

Procedeu-se a análise envoltória de dados através do software SIAD - Sistema Integrado de Apoio à Decisão (ANGULO MEZA et al., 2005) utilizando-se o modelo para avaliação de eficiência DEA-BCC com retornos variáveis de escala e orientação output (Quadro 1), construído a partir de uma combinação de cinco inputs e cinco outputs para cada DMU (Agência). Utilizou-se esse modelo, pois ele permite diferenciar as ineficiências técnicas daquelas de escala, identificando se as variações de escala identificadas são positivas, negativas ou constantes (COMELLI et al., 2017).

\section{ANÁLISE E DISCUSSÃO DOS RESULTADOS}

As 56 agências bancárias (DMU's) foram avaliadas a partir da abordagem da rentabilidade e, 22 foram consideradas eficientes (Tabela 1), pois foram aquelas que melhor combinaram o número de empregados e as despesas totais com a capacidade de geração de riqueza, representada pelo PIB do município, frente aos concorrentes presentes (inputs), obtendo assim, maiores resultados com menos insumos. Entre as demais agências, 12 delas apresentaram eficiência superior a 90\%, 17 agências apresentaram eficiência entre 70 e 90\%, 4 indicaram eficiência entre 50 e $70 \%$ e somente 1 agência apresentou resultado inferior a $50 \%$.

Tabela 1 - Eficiência das agências bancárias tradicionais analisadas

\begin{tabular}{cccccc}
\hline Agência & Eficiência & Agência & Eficiência & Agência & Eficiência \\
\hline AG1 & EFICIENTE & AG20 & $86,52 \%$ & AG40 & $84,21 \%$
\end{tabular}




\begin{tabular}{lccccc} 
AG2 & EFICIENTE & AG21 & $90,77 \%$ & AG41 & $87,35 \%$ \\
AG3 & EFICIENTE & AG22 & $77,17 \%$ & AG42 & EFICIENTE \\
AG4 & $90,83 \%$ & AG23 & EFICIENTE & AG43 & $84,56 \%$ \\
AG5 & EFICIENTE & AG24 & $90,16 \%$ & AG44 & $87,56 \%$ \\
AG6 & EFICIENTE & AG25 & EFICIENTE & AG45 & $91,21 \%$ \\
AG7 & $98,16 \%$ & AG26 & $79,51 \%$ & AG46 & EFICIENTE \\
AG8 & $94,07 \%$ & AG27 & $74,47 \%$ & $\mathbf{A G 4 7}$ & $96,29 \%$ \\
AG9 & $99,86 \%$ & AG28 & EFICIENTE & $\mathbf{A G 4 8}$ & $79,97 \%$ \\
AG10 & $92,81 \%$ & $\mathbf{A G 2 9}$ & $94,66 \%$ & $\mathbf{A G 4 9}$ & EFICIENTE \\
AG11 & $86,02 \%$ & $\mathbf{A G 3 0}$ & EFICIENTE & $\mathbf{A G 5 0}$ & EFICIENTE \\
AG12 & EFICIENTE & $\mathbf{A G 3 1}$ & $94,97 \%$ & $\mathbf{A G 5 1}$ & EFICIENTE \\
AG13 & EFICIENTE & $\mathbf{A G 3 2}$ & $68,82 \%$ & $\mathbf{A G 5 2}$ & EFICIENTE \\
AG14 & $71,76 \%$ & $\mathbf{A G 3 3}$ & $71,39 \%$ & $\mathbf{A G 5 3}$ & EFICIENTE \\
AG15 & $86,08 \%$ & $\mathbf{A G 3 4}$ & $90,84 \%$ & $\mathbf{A G 5 4}$ & $89,93 \%$ \\
AG16 & EFICIENTE & $\mathbf{A G 3 6}$ & EFICIENTE & $\mathbf{A G 5 5}$ & $62,96 \%$ \\
AG17 & $81,87 \%$ & $\mathbf{A G 3 7}$ & EFICIENTE & $\mathbf{A G 5 6}$ & $86,41 \%$ \\
AG18 & $85,58 \%$ & $\mathbf{A G 3 8}$ & $62,11 \%$ & $\mathbf{A G 5 7}$ & $44,15 \%$ \\
AG19 & $67,74 \%$ & $\mathbf{A G 3 9}$ & EFICIENTE & & \\
\hline
\end{tabular}

Fonte: elaborado pelos autores.

A partir dos resultados individuais de cada agência, identifica-se que as variáveis causadoras de ineficiência, mais encontradas, foram Receita Líquida (34 agências), número de concorrentes no município (26), margem de contribuição com tarifas (20) e número de empregados (18 agências), seguidas pelos demais apresentados na Tabela 2.

Esses resultados permitem inferir que todas as agências que apresentaram algum tipo de ineficiência tiveram também baixa receita líquida, ou excesso de insumos aplicados, ou baixo resultado dos produtos. Esses resultados corroboram os achados de Faria e Paula (2009) para os quais a baixa qualidade da gestão operacional, com elevados custos administrativos e empregados ociosos reduzem a receita líquida e, por consequência, a eficiência da unidade.

Tabela 2 - quantidade de agências com folga por variável

\begin{tabular}{lcc}
\hline \multicolumn{1}{c}{ Variável } & $\begin{array}{c}\text { Quantidade de agências } \\
\text { com folga (ineficientes) }\end{array}$ & $\begin{array}{c}\text { Representatividade } \\
\text { sobre as ineficientes }\end{array}$ \\
\hline Receita líquida (RLIQ) & 34 & $100 \%$ \\
Bancos que atuam no município (BCMUN) & 26 & $76 \%$ \\
Margem de contribuição Tarifas (MCTAR) & 20 & $59 \%$ \\
Total de Empregados (EMP) & 18 & $53 \%$ \\
Agências concorrentes (AGCON) & 17 & $50 \%$ \\
PIB do município (PIB) & 14 & $41 \%$ \\
Margem de contribuição produtos (MCPROD) & 13 & $38 \%$ \\
Despesas Totais (DPTOT) & 9 & $26 \%$ \\
Margem de contribuição média por cliente (MCMED) & 9 & $26 \%$ \\
Clientes (CLI) & 9 & $26 \%$ \\
\hline
\end{tabular}

Fonte: Dados da pesquisa.

Pontualmente, observa-se que a receita líquida é o principal indicador de folga, a qual é uma consequência da ineficiência na gestão dos demais insumos. Os achados de Camargo et al. (2004) argumentam nesse sentido, pois mostraram que aqueles bancos que pos- 
suem menor volume de ativos totais, conseguem uma gestão mais eficaz de seus insumos, obtendo melhor eficiência e, por consequência, maior rentabilidade.

Mesmo que a instituição estudada não utilize a Análise Envoltória de Dados para fins de mensuração de eficiência de suas unidades, esses resultados permitem avaliar suas estratégias quanto a captação de clientes e de atingimento de suas metas orçamentárias. Contudo, observa-se que várias agências possuem poucos concorrentes locais, o que contribui para o êxito na captação de clientes, principalmente por apresentar um elevado número de empregados por agência, outro fator de ineficiência.

Por outro lado, segundo Comelli, Kroenke e Hein (2017), o contexto econômico e o ambiente de negócios impactam a eficiência em relação à análise por intermediação, especialmente em casos em que existe folga na quantidade de empregados. Os resultados encontrados por tais autores se assemelham aos encontrados nesse estudo no que concerne a baixa eficiência de unidades com elevado número de empregados em municípios com PIB elevado e baixa concorrência de mercado.

Por fim, analisou-se individualmente a folga de cada variável das agências com eficiência inferior a $70 \%$ de modo que se pudesse indicar os itens prioritários para melhoria de desempenho, conforme Tabela 3.

Tabela 3 - Folga das agências com eficiência menor que $70 \%$ por variável

\begin{tabular}{ccccccccccc}
\hline & EMP & DPTOT & $\begin{array}{c}\text { PIB } \\
\text { R\$ }\end{array}$ & $\begin{array}{c}\text { BC } \\
\text { MUN }\end{array}$ & $\begin{array}{c}\text { AG } \\
\text { MUN }\end{array}$ & $\begin{array}{c}\text { MC PROD } \\
\text { R\$̦ }\end{array}$ & $\begin{array}{c}\text { MCTAR } \\
\text { R\$̦ }\end{array}$ & $\begin{array}{c}\text { RLIQ } \\
\text { R\$\$ }\end{array}$ & MCMED & CLI \\
\hline AG19 & - & $9.225,87$ & - & 0 & - & $10.930,49$ & - & $119.242,79$ & - & - \\
AG32 & - & $25.183,12$ & - & 0 & - & - & $2.632,50$ & $87.922,02$ & - & 162 \\
AG38 & - & - & - & 8 & 23 & $40.550,52$ & - & $123.696,48$ & - & - \\
AG55 & & - & - & - & - & - & - & $37.299,30$ & 3,98 & 252 \\
AG57 & & - & - & 7 & 13 & $15.865,63$ & - & $114.901,09$ & - & - \\
\hline
\end{tabular}

Fonte: Dados da pesquisa.

Pontualmente, observa-se que as agências AG19 e AG32 apresentam excessos de despesas totais, ocasionando folga com relação ao benchmark o que as torna ineficientes nesse quesito. Além disso, apresentam folgas em margem de contribuição de produtos e tarifas, respectivamente, o que indica a necessidade de ampliação do número de produtos por cliente (AG19) e de serviços bancários prestados por clientes (AG32). Além disso, a AG32 deve promover ações para prospecção de clientes, pois apresenta folga também nesse item.

A agência AG38 apresenta elevada folga com relação a margem de contribuição por produtos, além disso, especificamente nesse caso, a eficiência é ainda mais baixa, pois o número de bancos concorrentes e agências bancárias destes no município apresentam folga e, portanto, seu marketshare deveria ser ainda maior. Mesmo assim, apresenta folga também no item clientes. Evidencia-se que a estratégia para essa agência é a prospecção de novos clientes.

Por fim, para a AG57, por apresentar somente 44\% de eficiência, procedeu-se entrevista com o gestor da mesma para elucidar os fatos e constatou-se que a agência foi aberta há menos de 24 meses cujo payback estimado são 60 meses. Nesse caso, os indicadores de eficiência ficaram prejudicados, pois a agência inicia as atividades com uma estrutura de pessoal e com despesas fixas iniciais como aluguel, monitoramento e vigilância que inviabilizam o cálculo comparativo em relação as demais.

\section{CONSIDERAÇÕES FINAIS}


Diante do cenário de competitividade do mercado financeiro, acirrado recentemente pela entrada das fintechs, tornam-se necessárias reavaliações das eficiências das agências dos bancos tradicionais utilizando-se para isso métodos alternativos que identificam espaços para crescimento e melhoria da produtividade ou redução de gastos. Em consonância, realizou-se este estudo com utilização do método da Análise Envoltória de Dados para avaliação da eficiência das agências de um banco tradicional tendo como benchmark aquela de melhor desempenho.

Analisando-se a eficiência das agências pertencentes a um mesmo banco, comparando-as entre si, foi possível identificar que somente 22 agências $(40 \%)$ foram eficientes. As demais 34 agências apresentaram ineficiências e folga ou nos insumos (EMP, DPTOT, PIB, BCMUN, AGMUN) ou nos produtos (MCPROD, MCTAR, RLIQ, CLI, MCMED), das quais, 5 apresentaram eficiência inferior a $60 \%$.

A principal contribuição desse estudo é analisar a eficiência de unidades considerando também seu ambiente de negócios, isto é, o PIB do município em que se encontra e seus concorrentes (número de bancos e de agências). Desse modo, amplia-se o olhar sobre os insumos (inputs) da unidade não só para aqueles que já fazem parte da estrutura de despesas, como também o marketshare a ser explorado, em relação às demais analisadas.

Este estudo tem como limitação a data base dos dados, ou seja, o valor do PIB é 2014 e os demais dados são de 2017, o que pode prejudicar os resultados, contudo, não havia outra alternativa. Outra limitação é o fato de que os dados utilizados apresentam corte transversal e podem representar uma realidade pontual vivida pela agência. Além disso, foi utilizada somente uma instituição, logo, o indicador de eficiência é interno, não se podendo afirmar que as agências que resultaram eficientes nessa pesquisa apresentam eficiência no mercado.

Sugere-se que pesquisas futuras avancem no estudo da eficiência bancária avaliando mais de um banco e também as fintechs, com as mesmas variáveis apresentadas para que se possa obter índice de eficiência mais próximo da realidade de mercado. Por fim, sugere-se que seja utilizado um corte longitudinal para reduzir o viés causado por picos de prejuízos ou receitas que possam enviesar os resultados.

\section{Agradecimento}

Os autores agradecem o auxílio financeiro recebido do Conselho Nacional de Desenvolvimento Científico e Tecnológico (CNPq)

\section{REFERÊNCIAS BIBLIOGRÁFICAS}

[1] ABLANEDO-ROSAS, J. H., GAO, H., ZHENG, X., ALIDAEE, B., WANG, H. A study of the relative efficiency of Chinese ports. Expert systems, v. 27, n. 5 p. 349-362, 2010.

[2] ANGULO MEZA, L.; BIONDI NETO, L.; SOARES DE MELLO, J.C.C.B.; GOMES, E.G. ISYDS - Integrated System for Decision Support (SIAD - Sistema Integrado de Apoio à Decisão): a software package for data envelopment analysis model. Pesquisa Operacional, v. 25, (3), p. 493-503, 2005.

[3] ARAUJO, P. M. Q.; CARMONA, C. U. M. Eficiência de uma rede de agências bancárias utilizando O modelo Data Envelopment Analysis - DEA. Revista Produção Online. V. 2, n. 2, 2002.

[4] ASSAF NETO, A. Finanças Corporativas e Valor. São Paulo: Atlas, 2005. 
[5] . Mercado Financeiro. 3. ed. São Paulo: Atlas, 2000.

[6] BARBOSA, A. C. T. A. M.; MACEDO, M. A. S. O sistema bancário brasileiro: uma análise do desempenho através da DEA. Revista da ABCustos, v. 3, n. 3, p. 1-21, 2008.

[7] BERGER, A. N.; HUMPHREY, D. B. Efficiency of financial institutions: International survey and directions for future research. European Journal of Operational Research, v. 98, n. 2, p. 175-212, 1997.

[8] BERGER, A. N.; UDELL, G. F. The economics of small business finance: The roles of private equity and debt markets in the financial growth cicle. Journal of Banking \& Finance, v. 22, n. 6-8, 1998.

[9] CAMARGO, A. S.; MATIAS, A. B.; MERLO, E. M. Desempenho dos bancos comerciais e múltiplos de grande porte no Brasil. São Paulo: Mimeo, 2004.

[10] CARNEIRO, M.; PEREIRA, A. S. J.; MACORIS, L. S. Avaliação Da Eficiência Bancária Por Meio Da Abordagem De Intermediação: Uma Análise Comparativa De Instituições Financeiras Brasileiras. REAd-Revista Eletrônica de Administração, v. 22, n. 3, 2016.

[11] CARVALHO, C. C.; FORTE, S. H. A. C.; OLIVEIRA, O. V.; SALES, R. K. L. Mensuração da Capacidade de Governança Corporativa das Empresas Familiares para migração ao Novo Mercado da BM\&FBovespa. Revista de Administração FACES Journal, v. 15, n. 3, 2016.

[11] CASADO, Frank Leonardo. Análise envoltória de dados: conceitos, metodologia e estudo da arte na educação superior. Revista Sociais e Humanas, v. 20, n. 1, p. 59-71, 2007.

[12] CERETTA, P. S.; NIEDERAUER, C. A. P. Rentabilidade e eficiência no setor bancário brasileiro. Revista de Administração Contemporânea, v. 5, n. 3, 2001.

[13] COMELLI, Adriane; KROENKE, Adriana; HEIN, Nelson. Eficiência dos Bancos de capital aberto no Brasil: uma análise DEA. Anales de ASEPUMA, n. 25, p. 17, 2017.

[14] COOPER, W. W.; SEIFORD, L. M.; ZHU, J. Data envelopment analysis history, models and interpretations. Springer, 2011.

[15] DEBREU, G. The coefficient of resource utilization. Econometrica, Journal of the Econometric Society, v.19, no 3, 1951.

[16] DEPARTAMENTO INTERSINDICAL DE ESTATÍSTICA E ESTUDOS SOCIOECONÔMICOS - DIEESE. Desempenho dos Bancos 2016. 2017. Disponível em: https://www.dieese.org.br/desempenhodosbancos/2016/desemprenhoDosBancos2016.pdf. Acesso em 03 fev. 2018.

[17] DOUMPOS, M.; COHEN, S. Applying data envelopment analysis on accounting data to assess and optimize the efficiency of Greek local governments. Omega International Journal of Management Science, v. 46, p. 74-85, 2014. 
[18] FARIA, J. A. de; PAULA, L. F. Fusões e aquisições bancárias e a evolução da eficiência técnica dos maiores bancos privados no Brasil. In: XXXVII Encontro Nacional de Economia, ANPEC, Foz do Iguaçu, PR. Disponível em< https://www.researchgate.net/ profile/Luiz_Paula/publication/

241752039_fusoes_eaquisicoes_bancarias_e_aevolucao_da_eficienciatecnica_dos_maiores bancos_privados_no_brasil/links/00b49528 $\overline{8}$ ce $352 \mathrm{~b} 725 \mathrm{e} 000000 . \mathrm{pdf}>$. Acesso em $0 \overline{4} \mathrm{fev}$ 2018.

[19] FARREL, M. J. The measurement of produtive efficiency. J. Royal Statistical Society, v. 120, Part III, 253-290, 1957.

[20] FEBRABAN - Federação Brasileira de Bancos. Relatório Anual 2016. Disponível em: https://relatorioanual2016.febraban.org.br/pt/download/FEBRABAN_RA_16.pdf. Acesso em: 24 jan. 2018.

[21] FERREIRA, C. M. C; GOMES, A. P. Introdução a Análise Envoltória de Dados: Teoria, Modelos e Aplicações. 22ª ed. Viçosa/MG: Editora UFV, 2009.

[22] FINTECHLAB (2017). Retrospectiva Fintechlab 2017. Disponível em: $<$ http://fintechlab.com.br/index.php/2018/01/23/retrospectiva-fintechlab-mostra-mais-de-r-1-bi-de-investimentos-nas-fintechs-em-2017/>. Acesso em: 24 janeiro 2017.

[23] HASAN, M. Z.; KAMIL, A.A.; MUSTAFA, A.; BATEN, M.A. A Cobb Douglas Stochastic Frontier Model on Measuring Domestic Bank Efficiency in Malaysia. PLOS ONE, v. 7, n. 8, 2012.

[24] KOOPMANS, T. C. Efficient allocation of resources. Econometrica, v.19, n.1, 1951.

[25] KUUSSAARI, H. Productive Efficiency in Finnish Local Banking During 19851990. Helsinki: Suomen Pankin monistuskeskus, 1993.

[26] LIU, S. Measuring and categorizing technical efficiency and productivity change of commercial banks in Taiwan. Expert Systems with Applications, v. 37, n. 4, p. 2783-2789, 2010.

[27] MAXIMIANO, Antonio César Amaru. Introdução à administração. $8^{\circ}$ ed. São Paulo, Atlas, 2011.

[28] MENDONÇA, D. J.; SOUZA, J. A.; CAMPOS, R. S. Análise do desempenho dos maiores bancos brasileiros: um estudo com a análise envoltória de dados (DEA) aplicada a um conjunto de indicadores econômico-financeiros. Observatorio de la Economía Latinoamericana, n. 223, 2016.

[29] OLIVEIRA, T. L.; MARQUES, J. A. Relação Entre Grande Empresa E Startups: Principais Barreiras E Proposição De Ações De Curto Prazo. IV EMPRAD - Encontro dos Mestrados Profissionais em Administração - FEA/USP, 2017.

[30] SAHIN, G.; GOKDEMIR, L.; OZTURK, Dogan. Global Crisis and its Effect on Turkish Banking Sector: A Study with Data Envelopment Analysis. Procedia economics and finance, v. 38, p. 38-48, 2016. 
[31] SANJEEV, G. M. Data envelopment analysis (DEA) for measuring technical efficiency of banks. Vision, v. 10, n. 1, p. 13-27, 2006.

[32] SATHYE, M. Efficiency of banks in a developing economy: The case of India. European Journal of Operational Research, v. 148, n. 3, p. 662-671, 2003.

[33] SOUZA, M. F. A. S.; MACEDO, M. A. S. Análise de desempenho contábil-financeiro no setor bancário brasileiro por meio da aplicação da análise envoltória de dados (DEA). Revista BASE, v. 6, n. 2, p. 81-100, 2009.

[34] TAVARES, G. A Bibliography of Data Envelopment Analysis. Rutcor Research Report - RRR, 2002.

[35] THANASSOULIS, E. A comparision of regression analysis and data envelopment analysis as alternative methods for performance assessments. Journal of Operational Research Society, v. 44, n. 11, p. 1.128-1.144, 1993

[36] YANG, Z. Bank Branch Operating Efficiency: A DEA Approach. Proceedings of the International Multi Conference of Engineers and Computer Scientists, Vol II IMECS, 2009.

[37] ZHONG, Y.; ZHAO, J. The optimal model of oilfield development investment based on Data Envelopment Analysis. Petroleum, v. 2, n. 3, p. 307-312, 2016.

[38] ZHU, J. Multi-factor performance measure model with application to Fortune 500 companies. European Journal of Operational Research, v. 123, n. 1, p. 105-124, 2000. 